MODELING, IDENTIFICATION AND CONTROL, 1989, VOL. 10, NO. 3, 173-184

doi:10.4173/mic.1989.3.6

\title{
User experience with on-line predictive river flow regulation
}

\author{
B. FOSS $\dagger$, J. E. HAUG $\dagger$, J. ALNE $\ddagger$ and S. AAM $\S$
}

Keywords: River flow regulation, control, adaptive prediction, on-line operation.

A control strategy based on adaptive prediction and a receding horizon controller is used to regulate the river flow-rate in Suldalslagen in the Ulla-Førre production system on the south-west coast of Norway. The adaptive prediction of the unregulated inflow used precipitation data and flow-rate data from a stream in the catchment area. In addition, a recursive estimation algorithm is used to estimate the stationary gains of the parallel transfer function models which have the catchment area data as input and the unregulated inflow as output.

The system is implemented on an IBM-PC and has been in operation since summer 1986.

\section{Introduction}

The environmental impact of the regulation of rivers for hydro-electric power production has recently received increased attention. Important considerations for the public are the local climate and scenery, and the conditions for fishing and farming. These are the reasons why the authorities impose certain constraints on minimum flow in natural rivers when operating a power production system.

These stipulations may conflict with the obvious economic incentive of trying to save unnecessary water release to such rivers since this water can be used for power production. How much water to release from the traps depends on the dynamic characteristics of the river between the traps and the flow measurement mark as well as the local unregulated run-off to the river.

\section{Problem formulation}

In conjunction with Ulla-Førre power production system on the south-west coast of Norway, the authorities have imposed restrictive constraints on the flow rate in the river Suldalslagen. The flow measurement mark, which is checked by the authorities, is placed at the mouth of the river, approximately $20 \mathrm{~km}$ downstream of the traps. The fall is 67 metres and the time-delay from the traps to the measurement mark varies between three and six hours according to the flow rate of the river.

The minimum flow-rate restriction varies with the seasons from $100 \mathrm{~m}^{3} / \mathrm{s}$ in the autumn down to $15 \mathrm{~m}^{3} / \mathrm{s}$ in the winter. Very often, during the autumn, the variations in the local inflow to the river can be of the same magnitude as these restrictions, hence the

Received 1 June 1989

$\uparrow$ SINTEF, Division of Automatic Control, N-7034 Trondheim, Norway.

$\ddagger$ Norwegian State Power Board (Statkraft), N-4200 Sauda, Norway.

$\$$ The Norwegian Research Institute of Electricity Supply A/S (EFI), N-7034, Trondheim, Norway.

Reprinted with permission from the IEEE Power Engineering Society 1989 Winter Meeting, New York, NY, January 29-February 3, 1989. 
excitation of the process is very significant. The local inflow also varies rapidly with time since the surrounding area with its steep mountains has little degree of natural regulation. Therefore it is difficult to find an optimal control strategy which can regulate the traps so that the water flow at the said mark is kept at a minimum without violating the minimum flow constraint at any time.

Manual control of the traps from the dispatch centre has proved to be an inadequate solution to the control problem. There were two reasons for this. First, it was not possible to obtain satisfactory performance using manual control and second, this task demanded frequent attention. The company consequently installed a conventional controller to automatically control the river's flow rate. However, the performance of this controller was still not satisfactory. The solution was the development of an on-line controller, the subject of this paper.

\section{Model description}

It is convenient to divide the model of the river flow system into two main submodels which describe the dynamics of the river and the catchment area respectively.

\subsection{River model}

In earlier work (Fjeld, Foss and Aam (1983)) it has been shown that a 2nd order transfer function including a time-delay is an adequate model to describe the regulated river flow, $y_{r}$, at a certain mark subject to upstream changes in the flow rate.

$$
y_{r}(t)=\frac{B}{A} u(t-k)+\frac{C}{A} e(t)
$$

$u$ released flow from the traps

$e$ noise term

$A, B, C$ polynomials in the time-delay operator, $z^{-1}$

$k-1$ time-delay.

The time-constants and the time-delay vary as a function of the flow rate. This is exploited by a table look-up routine for calculating the river model parameters according to the flow rate. Our experience shows that excellent river dynamics matching can be accomplished using this technique. The fact that the stationary gain in the river model is always equal to one (because of the mass balance), is included in the look-up routine.

An advantage of this approach is that it omits the need for on-line parameter estimation of the river flow model since trials have shown that, in practice, it is impossible to estimate parameters in both the river and catchment area models.

\subsection{Modeling the unregulated flow}

The unregulated inflow, $y_{u}$, between the traps and the mark adds to the total river flow, $y$, at the mark,

$$
y(t)=y_{r}(t)+y_{u}(t) .
$$

Different run-off models with varying degrees of detail based on the description of the natural physical processes have been developed during the last twenty years. In Norway the so-called HBV-model (Fjeld and Aam (1980)) is used with a prediction horizon of between one and a hundred days with a time resolution of one day. For the 
matter in hand, we only need to predict the unregulated inflow with a horizon of 3 to 6 hours. The model is not used during the winter season. Consequently, a simpler transfer function model is sufficient to describe the time-varying part, $y_{u}^{v}$ of the run-off process as shown below.

$$
y_{u}^{v}(t)=\frac{D_{i}}{A_{i}} v_{i}\left(t-k_{i}\right)
$$

$v_{i}$ model input no. $i$, e.g., precipitation data.

$D_{i}, A_{i}$ polynomials in the time-delay operator, $z^{-1}$.

$k_{i}-1$ time-delay.

The noise term is omitted as it is assumed that the total noise is accumulated in the noise term in (1).

The rapidly time-varying part of the inflow is caused by precipitation.

The major problem in identifying the above model is the representativity issue. If, for instance, $v_{i}$ represents the precipitation at one point in the catchment area, the stationary gain, in particular, between the precipitation and the unregulated inflow varies because of local variations in the precipitation. By using the flow rate in a small stream in the catchment area as input to the model in (3) one reduces the representativity problem since the flow rate in such a stream is accumulated from the local catchment area of the stream. The advantage of utilizing precipitation data, however, is the fact that the time-delay, $k_{1}$, is larger than in the case of flow-rate data from a small stream. This is important since it is essential to get an early warning of an increase in the unregulated infiow because of the large time-delay in the river model. In order to take advantage of both types of data, we use three parallel models of the type shown above, (3), since data from two different precipitation measurement locations and from one small stream in the catchment area are available on-line. By using parallel models like that shown in the upper part of Fig. 1, in addition to on-line estimation of the stationary gain of each of the models, we reduce the representativity problem to a minimum. Trials show that second order transfer functions are adequate in all three transfer function models.

Apart from the unregulated inflow caused by precipitation there is a slowly varying inflow, $y_{u}^{s}$, due to the average water content in the soil of the catchment area, hence the total run-off is modeled by

$$
y_{u}(t)=y_{u}^{v}(t)+y_{u}^{s}(t)
$$

\section{Controller}

\subsection{Predictive control strategy}

As mentioned, a common factor in river flow systems is relatively long time delays. This calls for some kind of predictive control strategy, i.e., the control input is calculated according to a prediction of the river flow at the mark. This can be stated as follows:

$$
y^{*}(t+k \mid t)=r(t+k)
$$

$y^{*}(t+k \mid t)$ is a $k$-step ahead predictor of the system's output, i.e., information until sample $t$ is used to predict the output, $k$ time-steps ahead. $r$ is the set-point.

The controller is based on a modification of the generalized minimum variance (GMV) controller, the receding horizon (RH) controller. The theory is outlined in 
Appendix A. This algorithm has been chosen for its robustness with respect to variation in the time-delay. The criterion which is minimized, cf. (A.9) is:

$$
J=E\left[\left(P y\left(t+k^{\prime}\right)-r\left(t+k^{\prime}\right)+Q u(t)\right)^{2}\right]
$$

$E(\quad)$ is the expectation operator, while $P$ and $Q$ are polynomials (or transfer functions) in the delay operator $z^{-1} \cdot k^{\prime}$ is the prediction horizon, which has to be equal to or longer than the system's time delay between the control input and the flow rate at the measurement mark.

Besides the notion of predictive control, satisfactory prediction of the unregulated inflow based on the model discussed earlier is imperative for the success of the controller. This is because of the extremely large and rapid variations of the inflow in the river Suldalslågen.

The criterion in (6) may be modified by only weighting the regulated flow by $P$. This means that the disturbance from the unregulated flow is separated from the regulated flow in the criterion.

$$
\begin{gathered}
J=E\left[\left(P y_{r}\left(t+k^{\prime}\right)-r_{v}\left(t+k^{\prime}\right)+Q u(t)\right)^{2}\right] \\
r_{v}\left(t+k^{\prime}\right)=r\left(t+k^{\prime}\right)-y_{u}^{*}\left(t+k^{\prime} \mid t\right)
\end{gathered}
$$

$r_{v}$ is an artificial reference signal for the part of the river flow that originates from being released through the trcps.

The total structure of the controller is shown in Fig. 1. The lower part (below the broken line) contains the RH-controller. Both the ballistically-calculated regulated flow-rate and the artificial reference signal are input. The structure of the unregulated inflow predictor is shown above the broken line. This predictor uses an estimate of the unregulated inflow (by subtracting the calculated regulated flow rate from the measured total flow rate). In addition two precipitation measurements and flowrate data from a stream are used as input. In the following the predictor is discussed in detail, see Fig. 1 for an overview of the predictor.

\subsection{Run-off prediction}

The run-off prediction is divided into slowly and rapidly varying parts.

Prediction of the slowly varying part, $y_{w}^{s}$, is simple since it does not significantly change during the prediction horizon (3-6 hours)

$$
y_{u}^{s^{*}}\left(t+k^{\prime} \mid t\right)=y_{u}^{s}(t)
$$

There is a problem, however, in deciding on $y_{u}^{s}(t)$, i.e., the actual stationary inflow. This is done as follows:

When the river flow is approximately stationary, the estimated stationary part of the river flow is computed according to:

$$
y^{s}(t)=T^{\prime} y^{s}(t-1)+\left(1-T^{\prime}\right) y(t), \quad 0<T^{\prime}<1
$$

The time-constant, of this filter has been selected to be 24 hours. When the river flow exhibits significant variations, the stationary part of the river flow is not updated. The test for discriminating between a stationary and non-stationary condition is given by:

Definition: Stationary river flow condition

$$
\begin{aligned}
\mid\left(y_{u}(t)-y_{u}(t-1)|<\gamma \wedge| y_{u}(t-1)-y_{u}(t-2) \mid<\gamma\right. \\
\gamma=0.5^{3} \mathrm{~m} / \mathrm{sec} .
\end{aligned}
$$




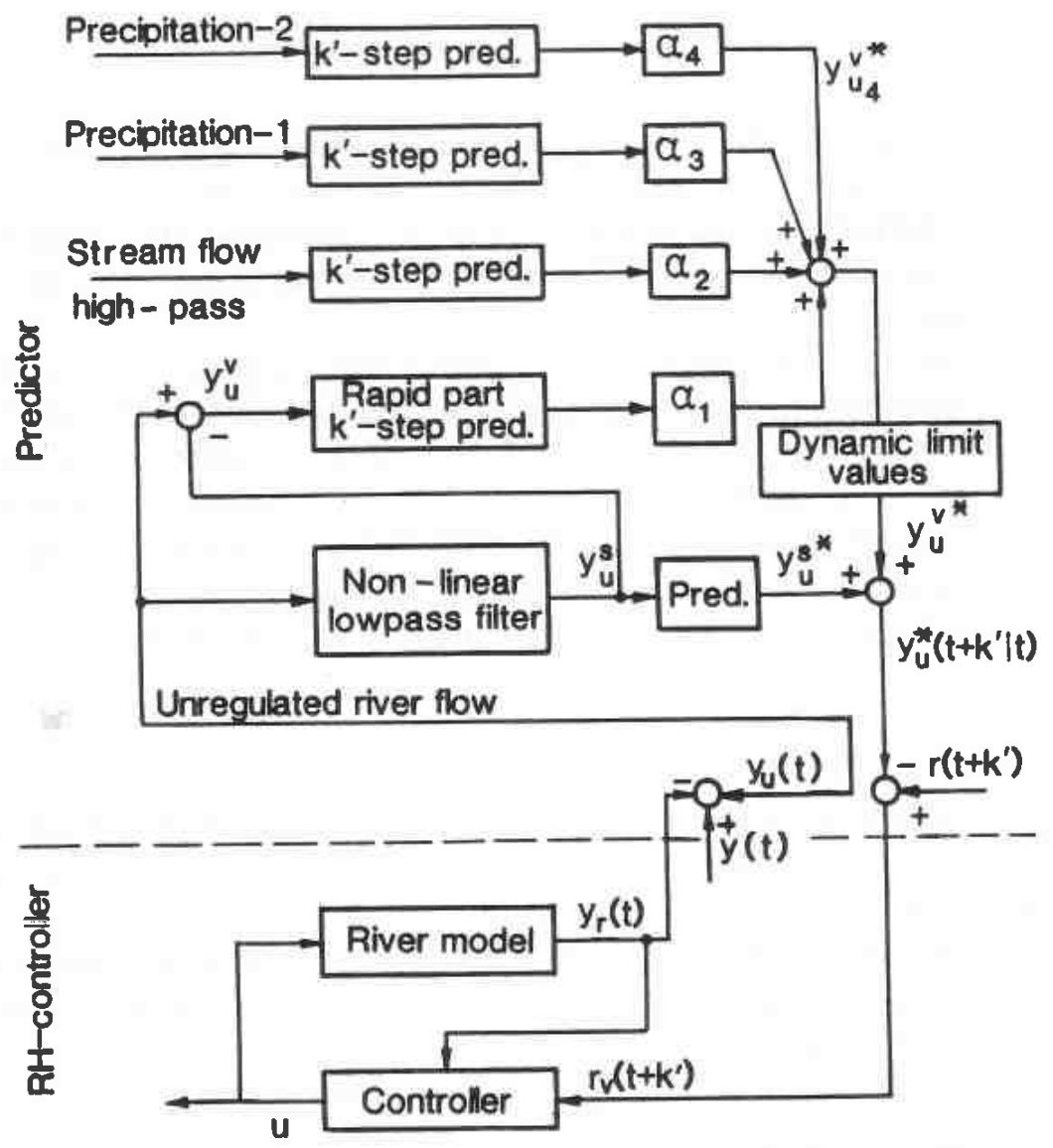

Figure 1. A schematic view of the controller divided into two main parts; the predictor and the receding horizon $(\mathrm{RH})$ controller parts.

In some cases the stationary component, $y_{u}^{s}$, tends to become too large, for instance, this sometimes happens shortly after heavy rainfall. This is caused by the rather long time constant, in the filter, cf. (10). These situations are handled in the following way:

$$
y_{u}^{s}(t)>y_{u}(t)=>y_{u}^{s}(t)=y_{u}(t)
$$

This last restriction overrides the two above conditions.

The rapidly varying inflow, $y_{u}^{v}$, is, in essence, computed by weighting the prediction from four different predictors, hẹnce we have:

$$
\begin{gathered}
y_{u}^{v^{*}}\left(t+k^{\prime} \mid t\right)=\sum_{i=1}^{4} \alpha_{i} y_{u_{i}}^{v^{*}}\left(t+k^{\prime} \mid t\right) \\
\sum_{i=1}^{4} \alpha_{i}=1, \quad \alpha_{i} \geqslant 0
\end{gathered}
$$

Let us now discuss the four predictors. They are all based on (3).

The first prediction is different from the three others in that no measurement is utilized in the catchment area, only the present value of the rapidly time-varying inflow. Hence, the input to this model, $v_{1}$, is an artificial input associated with the rainfall.

$$
\text { Model } \quad y_{u_{1}}^{v}(t)=\frac{D_{1}}{A_{1}} v_{1}(t)
$$




$$
\text { Predictor } \quad y_{u_{1}}^{v^{*}}\left(t+k^{\prime} \mid t\right)=\frac{D_{1}}{A_{1}} v_{1}\left(t+k^{\prime}\right)
$$

$D_{1} / A_{1}$ is a fixed second order transfer function. The prediction assumes that future values of $v_{1}$, i.e. $v_{1}(t+1), \ldots, v_{1}\left(t+k^{\prime}\right)$, are zero or equal to $v_{1}(\mathrm{t})$.

This choice depends on the consistency of the catchment area data. If they all suggest heavy rainfall future values of $v_{1}$ are chosen equal to $v_{1}(t)$. On the whole, the prediction is conservative.

The reason for including this predictor is primarily to take care of situations where there is little consistency in the predictions from the other three predictors because of local variations in the catchment area. In this case one has to opt for a conservative prediction of the inflow due to the restrictive minimum constraints. In addition Fig. 1 shows how this predictor provides the only feedback loop in the prediction part (apart from the very slow parameter estimation loops).

Predictions using the model with measured inputs are given by

$$
y_{u_{i}}^{v^{*}}\left(t+k^{\prime} \mid t\right)=\frac{D_{i}}{A_{i}} v_{i}\left(t+k^{\prime}-k_{i}\right), \quad i=2,3,4
$$

For the precipitation inputs $k^{\prime}<k_{i}$, the prediction is straightforward. In the case of the stream data we have $1<k_{i}<k^{\prime}$. Here future stream data is assumed to decay according to a time constant.

The stream flow rate is high-pass filtered to eliminate the stationary value of the flow rate. This is done for the same reasons as the division of the inflow into slowly timevarying and rapidly time-varying parts.

The predictor also includes a certain amount of logic.

The weighting factors, $\alpha_{1}, \ldots, \alpha_{4}$, are chosen according to a set of prespecified rules, cf. Appendix B. These values depend mainly on the number of active measurement devices at a given moment, hence they are used to adapt to situations where one or several parts of the measurement data are absent.

The dynamic limits of the predicted inflow (see Fig. 1) are computed by the use of the rapidly varying inflow, $y_{u}^{v}(t)$. The minimum limit is, for instance, computed by assuming future inputs $v_{1}$ equal to zero and choosing the time constant equal to its minimum value in the prediction equation, hence a smaller value than the value used in Eqn. (15).

The stationary gain is estimated in the models in (16) using the well known least squares recursive algorithm. The computed rapidly time-varying inflow, $y_{u}^{v}(t)$, is used as the measured input in these cases.

\section{Implementation}

The control system was implemented in summer 1986 on an IBM-PC located near the traps. On-line precipitation and stream data are read every 15 mins. while a new control input is computed every half hour. The computed control signal, i.e., the release from the traps, is also communicated as a setpoint every half hour to a flow control loop which regulate the traps.

Information regarding the control loop is available at the dispatch centre via a data communication link.

The reliability of the hardware has been satisfactory during its first two years of operation. 


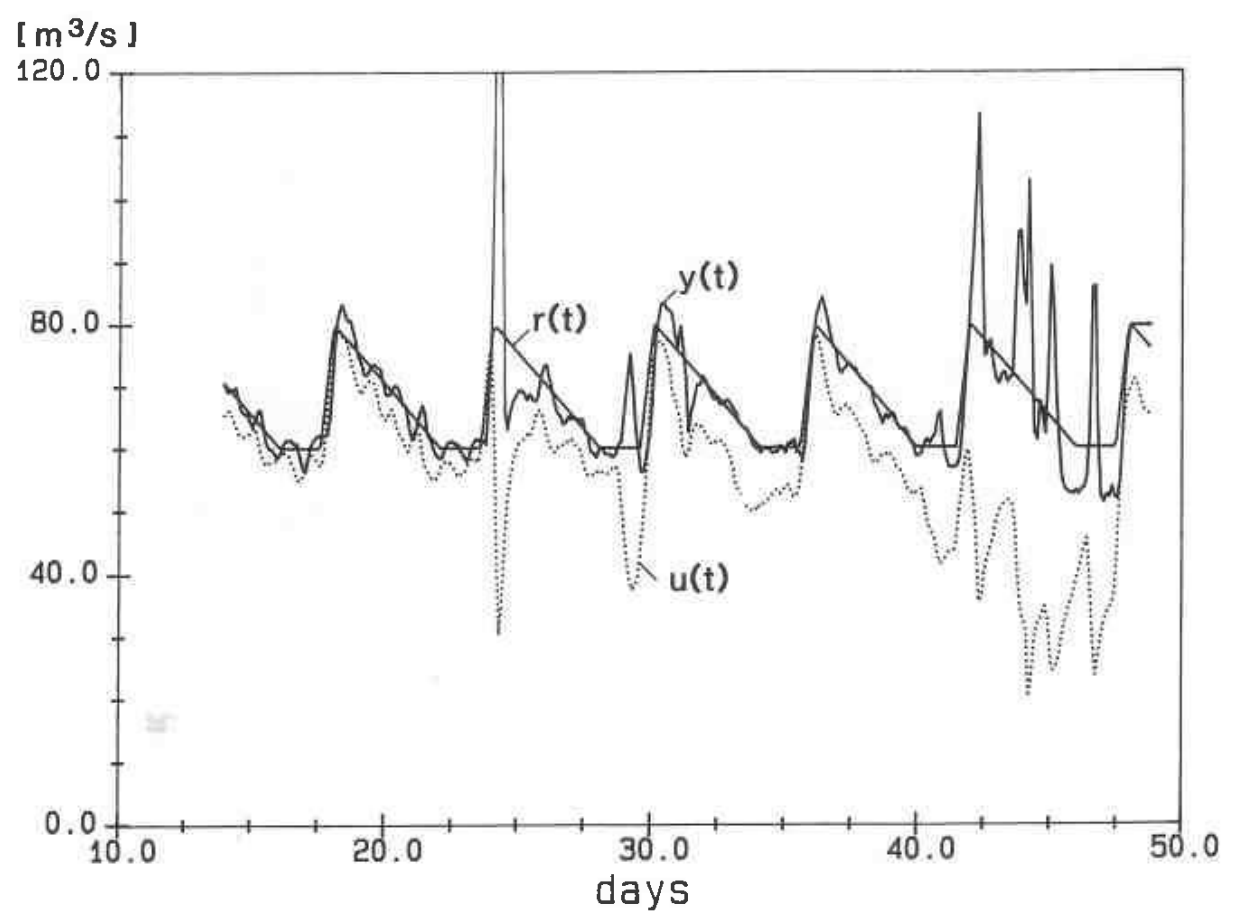

Figure 2. Controller performance during a five week period in September-October 1987.

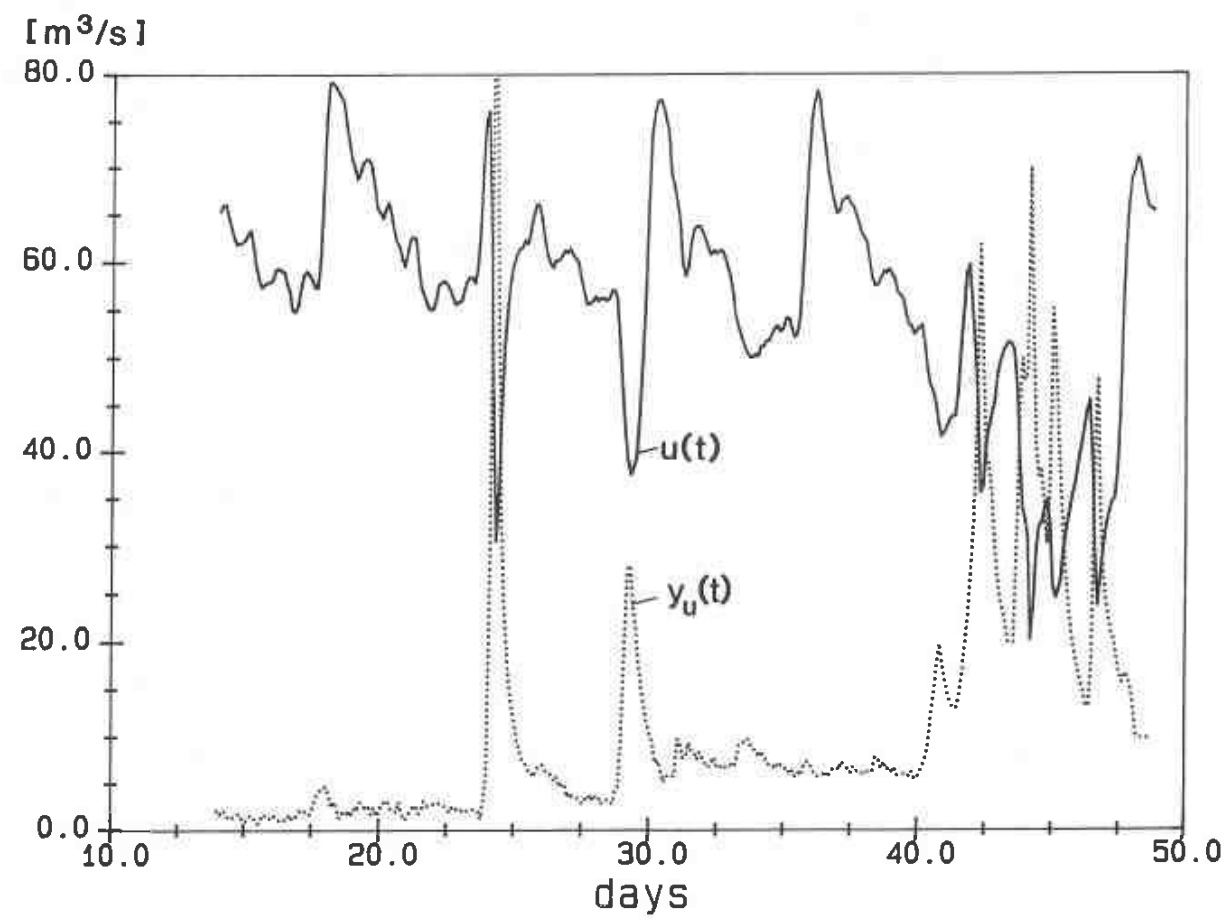

Figure 3. A comparison between the unregulated inflow and the release from the traps during the same time period as in Figure 2. 


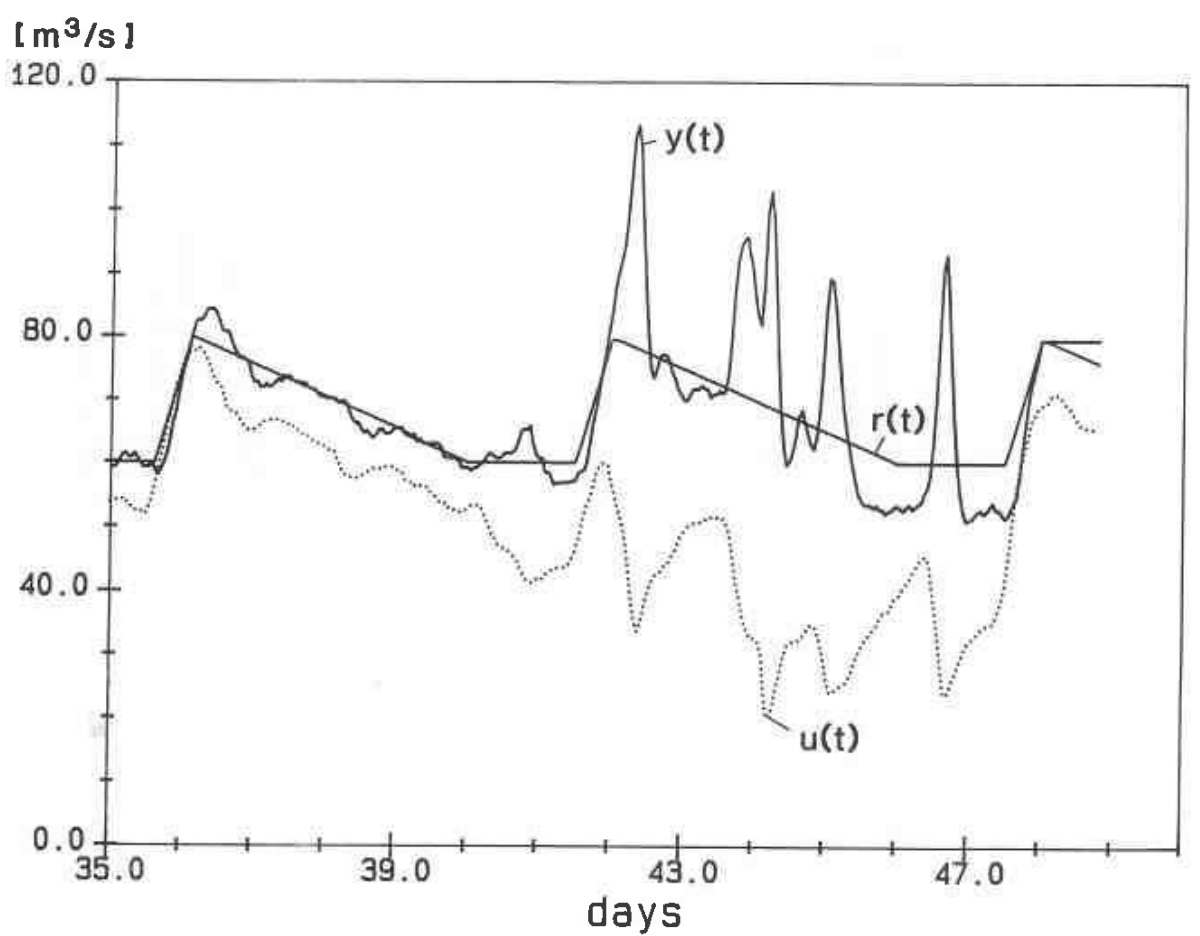

Figure 4. The controller performance during a period with heavy rainfall in October 1987 (the end of the time period shown in Figs. 2 and 3).

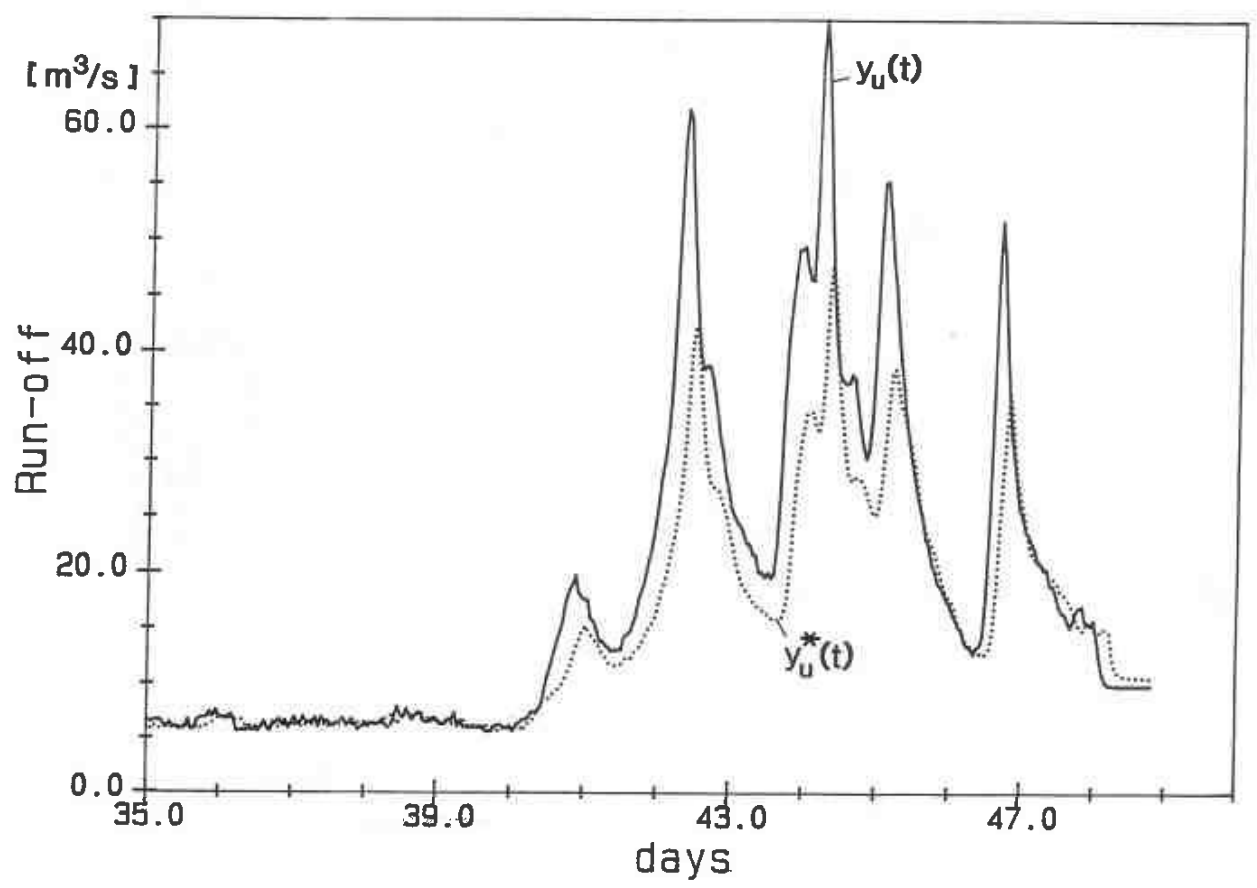

Figure 5. A comparison between unregulated inflow, $y_{u}(t)$, and its prediction, $y_{u}^{*}(t)$, during the period of heavy rainfall (Fig. 4). 


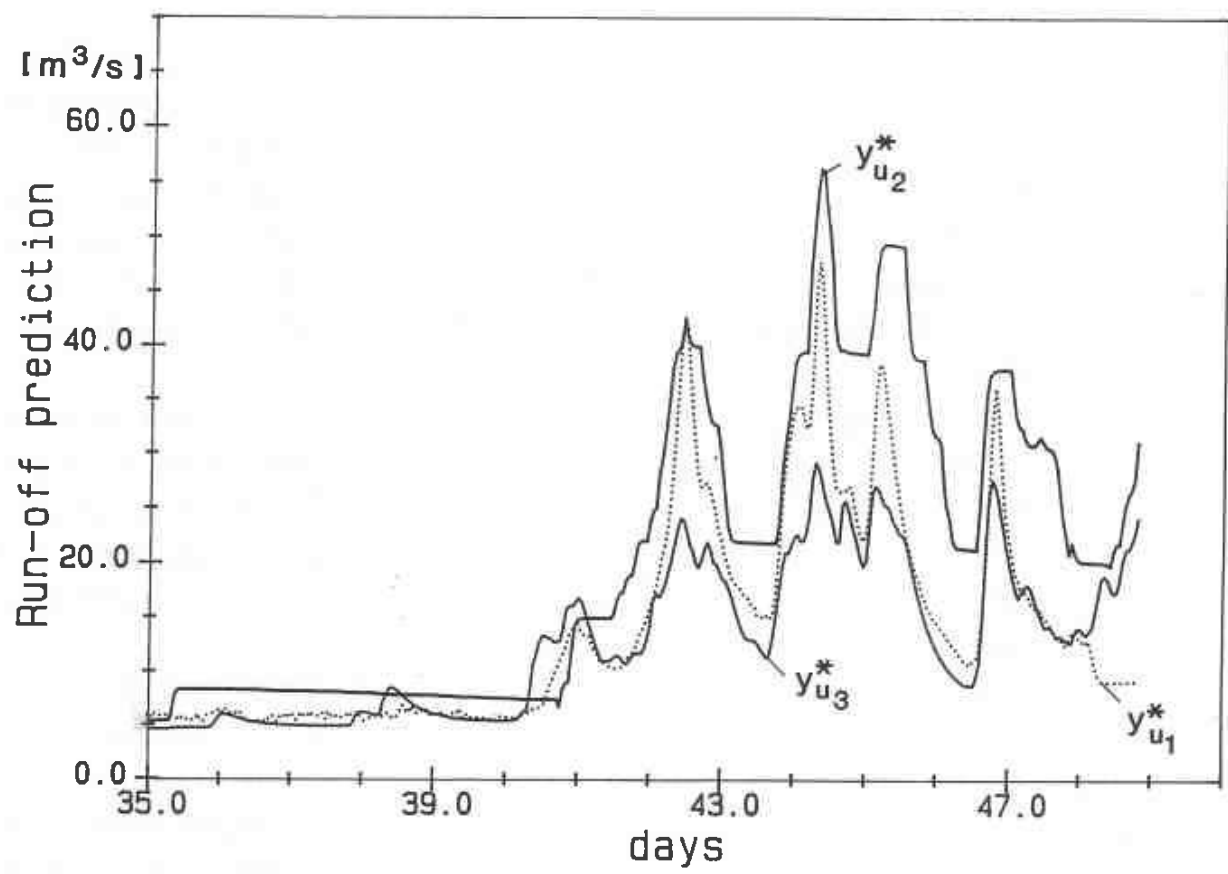

Figure 6. A comparison between the different predictors during the period of heavy rainfall (Fig. 4).

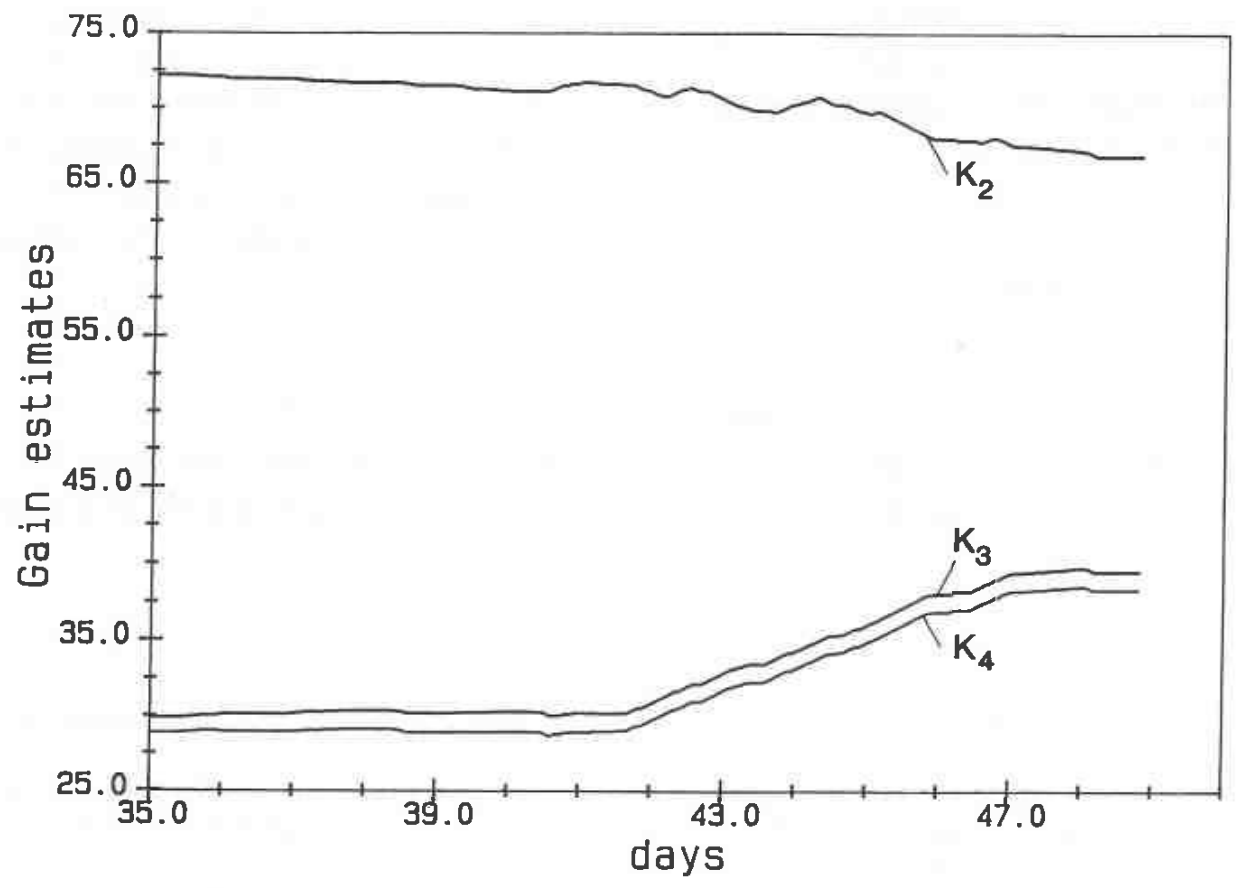

Figure 7. The stationary gain estimates, $K_{2}$ and $K_{3}$ and $K_{4}$ in the stream model and in the precipitation models, respectively, during the period of heavy rainfall (Fig. 4). 


\section{Results}

Some results will be presented in this section. Figures 2 and 3 show the behaviour of some important variables during a five week period (September-October 1987). The reference signal changes according to a prespecified cyclic function during this timeperiod. This has been done to improve the conditions for fishing. In periods with small variation in the unregulated inflow the controller performs well. It is however obvious that it is impossible to fully compensate for the extremely large and rapid changes in the inflow due to rainfall.

A part of the five week period is shown in Figs. 4-7. In Fig. 4 the flow-rate keeps roughly $10 \mathrm{~m}^{3} / \mathrm{s}$ below the reference signal for two one-day periods (days 46-48). The reason for this is that the authorities accepts that the minimum constraint is calculated according to the average value over a five day period. This has been exploited in the controller by modifying the reference signal accordingly, since this contributes to minimizing the release of water.

The inflow exhibits large variations. Figure 5 shows that the prediction of the inflow is somewhat conservative. Figure 6 shows that it is important to include several measurements in the catchment area since the predictions vary significantly.

The parameter estimates are stated in Fig. 7. The gains in the precipitation models increase towards the end of the time-period. This is logical when viewing the rather low predictions from these models during this time-period, see Figs. 5 and 6.

\section{Conclusions}

This paper describes a control algorithm for river flow regulation which uses an adaptive prediction model for the unregulated run-off to the river. The prediction model can take both measurements of precipitation and flow in streams in the local catchment area into consideration. Because the river flow is a process with a relatively long time delay, this controller has significantly better performance than a traditional PID controller. This improvement saves a quantity of water worth about $\$ 50000$ per annum when it is used for electric power production in the Hylen power station.

The controller has shown good performance during its first two years of operation, minor modifications have been made during this time. The reliability of the hardware has been satisfactory.

\section{ACKNOWLEDGMENTS}

This project was sponsored by the Royal Norwegian Council for Scientific and Industrial Research, the Water System Management Association and STATKRAFT.

\section{REFERENCES}

FJELD, M., Foss, B. and AAM, S. (1983). Regulation of river water level by adaptive control of a reservoir trap, American Control Conference, San Francisco.

FJELD, M. and AAM, S. (1980). An implementation of estimation techniques to a hydrological model for prediction of runoff to a hydroelectric power station, IEEE Trans. Automatic Control, 25, 151-163.

Clarke, D. W. and Gawthrop, B. A. (1979). Self-tuning control, Proc. IEE, 126, 633-640.

YDSTIE, B. E. (1982). Robust adaptive control of chemical processes, Ph.D. Thesis, Imperial College, London. 


\section{Appendix A}

\section{Receding horizon control}

The controller is based on Clarke and Gawthorp (1979) and Ydstie (1982).

Given an ARMAX-model (the delay operator $z^{-1}$ is omitted)

$$
y(t)=\frac{B}{A} u(t-k)+\frac{C}{A} e(t)
$$

it may be rewritten as:

$$
\Phi(t+k)=P y(t+k)=\frac{B P}{A} u(t)+\frac{C P}{A} e(t)
$$

$P$ is a polynomial.

The quotient $\frac{C P}{A}$ is now split

$$
\frac{C P}{A}=E+z^{-k} \frac{F}{A} \Rightarrow C P=A E+z^{-k} F
$$

An optimal predictor of $\Phi$ (in the minimum variance sense) may be formulated using (A. 1) -(A. 3).

$$
\Phi^{*}(t+k \mid t)=\frac{F}{C} y(t)+\frac{B E}{C} u(t)
$$

The generalized minimum variance controller minimizes the following criterion

$$
J=E\left[(\Phi(t+k)-r(t+k)+Q u(t))^{2}\right]
$$

$E$ is the expectation operator.

$Q$ is a polynomial in the time-delay operator $z^{-1}$.

The optimal predictor, (A.4), is used to calculate the control input $u(t)$ by minimizing (A. 5).

$$
\Phi^{*}(t+k \mid t)-r(t+k)+Q u(t)=0
$$

Given an ARMAX-model the controller calculations may be divided into two steps. First the Diophantine equation, (A.3), is solved in order to calculate the $E$ and $F$ polynomials. Secondly the control input is calculated using Eqn. (A. 6). It should be mentioned that $P$ and $Q$, in general may be transfer functions.

The prediction horizon may be increased, (A.4) becomes:

$$
\Phi^{*}(t+k \mid t)=\frac{F^{\prime}}{C} y(t)+\frac{B E^{\prime}}{C} u\left(t+k^{\prime}-k\right), \quad k^{\prime} \geqslant k
$$

$E^{\prime}$ and $F^{\prime}$ are different from $E$ and $F$ in (A. 4) if $k>k^{\prime}$, since they are calculated using $k^{\prime}$ instead of $k$ in the Diophantine equation (A.3).

The control input is calculated assuming,

$$
u\left(t+k^{\prime}-k\right)=\ldots=u(t), \quad k^{\prime} \geqslant k
$$

hence assuming the control input is constant in the next $\left(k^{\prime}-k\right)$ time steps.

(A. 5) and (A.6) are slightly changed

$$
J=E\left[\left(\Phi\left(t+k^{\prime}\right)-r\left(t+k^{\prime}\right)+Q u(t)\right)^{2}\right]
$$




$$
\Phi^{*}\left(t+k^{\prime} \mid t\right)-r\left(t+k^{\prime}\right)+Q u(t)=0
$$

Given an ARMAX-model the controller uses (A. 3) (with $k$ replaced by $k^{\prime}$ ), (A. 7), (A. 8) and (A. 10) to calculate the control input.

\section{Appendix B}

Rules for weighting factors

The weighting factors $\alpha_{1}, \ldots, \alpha_{4}$ are defined in the upper part of Fig. 1. They determine the prediction of the rapidly time-varing part of the inflow, see (13). They are chosen according to the following rules:

During normal conditions the weightings from (15), the stress flow data and precipitation data respectively are approximately equal, i.e., $\alpha_{1}=\alpha_{2}=\alpha_{3}+\alpha_{4}$. If the predictions from the models, which utilize precipitation data as their input, are inconsistent $\alpha_{3}$ and $\alpha_{4}$ are reduced compared to $\alpha_{1}$ and $\alpha_{2}$.

The precipitation model which predicts the lowest inflow value is always given higher confidence, due to the restrictive minimum flow constraints. 